\title{
Betti numbers of lex ideals over some Macaulay-Lex rings
}

\author{
Jeff Mermin • Satoshi Murai
}

Received: 2 August 2008 / Accepted: 15 June 2009 / Published online: 27 June 2009

(C) Springer Science+Business Media, LLC 2009

\begin{abstract}
Let $A=K\left[x_{1}, \ldots, x_{n}\right]$ be a polynomial ring over a field $K$ and $M$ a monomial ideal of $A$. The quotient ring $R=A / M$ is said to be Macaulay-Lex if every Hilbert function of a homogeneous ideal of $R$ is attained by a lex ideal. In this paper, we introduce some new Macaulay-Lex rings and study the Betti numbers of lex ideals of those rings. In particular, we prove a refinement of the Frankl-Füredi-Kalai Theorem which characterizes the face vectors of colored complexes. Additionally, we disprove a conjecture of Mermin and Peeva that lex-plus- $M$ ideals have maximal Betti numbers when $A / M$ is Macaulay-Lex.
\end{abstract}

Keywords Lex ideals · Graded Betti numbers · Hilbert functions · Colored simplicial complexes

\section{Introduction}

The Hilbert function is an important invariant of homogeneous ideals of a polynomial ring $A=K\left[x_{1}, \ldots, x_{n}\right]$ over a field $K$, studied in commutative algebra, algebraic geometry and combinatorics. One of the central results in the study of Hilbert functions is Macaulay's Theorem [14], which characterizes the Hilbert functions of homogeneous ideals of $A$ in terms of lex ideals. In the 1990's, a remarkable extension

The first author is supported by an NSF Postdoctoral fellowship (award No. DMS-0703625). The second author is supported by JSPS Research Fellowships for Young Scientists.

J. Mermin

Department of Mathematics, University of Kansas, Lawrence, KS 66045, USA

e-mail: mermin@math.ku.edu

S. Murai $(\bowtie)$

Department of Mathematics, Graduate School of Science, Kyoto University, Sakyou-ku,

Kyoto 606-8502, Japan

e-mail: murai@math.kyoto-u.ac.jp 
of Macaulay's Theorem was proved by Bigatti [2], Hulett [12] and Pardue [23]. They proved that lex ideals have the greatest graded Betti numbers among all homogeneous ideals having the same Hilbert function. In this paper, we introduce a class of monomial ideals $M$ such that Macaulay's Theorem holds for the quotient ring $A / M$, and study the graded Betti numbers of lex ideals of those rings.

Let $M$ be a monomial ideal of $A$ and set $R=A / M$. Recall that the Hilbert function $\operatorname{Hilb}(N)(-): \mathbb{Z} \rightarrow \mathbb{Z}$ of a finitely generated graded $R$-module $N$ is the function defined by

$$
\operatorname{Hilb}(N)(d)=\operatorname{dim}_{K} N_{d},
$$

where $N_{d}$ is the homogeneous component of degree $d$ of $N$. A set $W$ of monomials of $R$ is said to be a lex-segment if, for all monomials $u, v \in R$ of the same degree, $u \in W$ and $v>_{\text {lex }} u$ imply $v \in W$, where $>_{\text {lex }}$ is the degree lexicographic order. We say that a monomial ideal $I$ of $R$ is a lex ideal if the set of monomials in $I$ is a lexsegment. The ring $R$ is said to be Macaulay-Lex if, for any homogeneous ideal $J$ of $R$, there exists a lex ideal of $R$ having the same Hilbert function as $J$.

By Macaulay's Theorem [14], the polynomial ring $A$ itself is Macaulay-Lex. A famous class of Macaulay-Lex rings is the Clements-Lindström rings [4] $R=$ $A /\left(x_{1}^{a_{1}}, \ldots, x_{n}^{a_{n}}\right)$, where $1 \leq a_{1} \leq \cdots \leq a_{n}$ are integers or $\infty$. The notion of Macaulay-Lex rings was introduced in [17], and basic properties of Macaulay-Lex rings were established in $[17,18]$. A fundamental problem about Macaulay-Lex rings is the following.

Problem 1.1 (Mermin-Peeva) Find classes of monomial ideals $M$ of $A$ such that $A / M$ is Macaulay-Lex.

A homogeneous ideal $I$ of $A$ is said to be homogeneous-plus- $M$ (resp. lex-plus- $M$ ) if there exists a homogeneous (resp. lex) ideal $J$ such that $I=J+M$. Clearly, $A / M$ is Macaulay-Lex if and only if, for any homogeneous-plus- $M$ ideal $I$, there exists a lex-plus- $M$ ideal having the same Hilbert function as $I$. Inspired by the BigattiHulett-Pardue Theorem as well as Evans' Lex-plus-powers Conjecture [7], Mermin and Peeva made the following conjecture in [18].

Conjecture 1.2 (Mermin-Peeva) Suppose that $R=A / M$ is Macaulay-Lex.

(1) Every lex ideal $L$ of $R$ has the greatest graded Betti numbers among all homogeneous ideals of $R$ having the same Hilbert function as $L$.

(2) Every lex-plus- $M$ ideal $L$ of $A$ has the greatest graded Betti numbers among all homogeneous-plus- $M$ ideals of $A$ having the same Hilbert function as $L$.

Note that (1) considers infinite free resolutions, while (2) considers finite free resolutions. Conjecture 1.2 has been well studied for Clements-Lindström rings $R=A /\left(x_{1}^{a_{1}}, \ldots, x_{n}^{a_{n}}\right)$. In this special case, Conjecture 1.2(2) was proved in a series of papers [16, 19, 20], and Conjecture 1.2(1) was proved in [22] when the characteristic of $K$ is 0 . On the other hand, little is known for other Macaulay-Lex rings. In this paper we consider the following rings. 
Definition 1.3 Let $V=\bigcup_{j=1}^{r} V_{j}$ be a set of variables with $V_{j}=\left\{x_{j, 1}, \ldots, x_{j, n_{j}}\right\}$, where $n_{1} \geq n_{2} \geq \cdots \geq n_{r}$. Denote by $S=K[V]$ the polynomial ring over $K$ with the set of variables $V$. We will work with the lexicographic order $>_{\text {lex }}$ on $S$ induced by the ordering of the variables defined by $x_{k, \ell}>x_{k^{\prime}, \ell^{\prime}}$ if $\ell>\ell^{\prime}$ or $\ell=\ell^{\prime}$ and $k<k^{\prime}$. Let $Q=\sum_{j=1}^{r}\left(x_{j, 1}, \ldots, x_{j, n_{j}}\right)^{2} \subset S$. We call the ring $R=S / Q$ an $r$-colored squarefree ring of type $\left(n_{1}, \ldots, n_{r}\right)$.

We say that a quotient ring $R=A / M$ admits ideals with maximal Betti numbers over $A$ if, whenever $H$ is the Hilbert function of some homogeneous-plus- $M$ ideal of $A$, there exists a homogeneous-plus- $M$ ideal $L$ with Hilbert function $H$ such that $\beta_{i, j}(L) \geq \beta_{i, j}(I)$ for all $i, j$ and for all homogeneous-plus- $M$ ideals $I$ with Hilbert function $H$, where $\beta_{i, j}(J)$ are the graded Betti numbers of an ideal $J$ of $A$. Thus Conjecture 1.2(2) states that Macaulay-Lex rings admit ideals with maximal Betti numbers. The main results of this paper are the following.

- Colored squarefree rings are Macaulay-Lex.

- An $r$-colored squarefree ring of type $\left(n_{1}, \ldots, n_{r}\right)$ does not admit ideals with maximal Betti numbers over $S$ if $r=2$ and $n_{2} \geq 4$ or if $r \geq 3$ and $n_{r} \geq 3$.

- A computation of the graded Betti numbers of Borel ideals over $R$.

In particular, the second result disproves Conjecture 1.2(2).

The first result is inspired by the Frankl-Füredi-Kalai Theorem [8], which characterizes face vectors of colored simplicial complexes. Indeed, if $n_{1}=\cdots=n_{r}$ then the result is equivalent to the Frankl-Füredi-Kalai Theorem (see Remark 2.12 for details). Our proof is different from the original proof of the Frankl-Füredi-Kalai Theorem, but similar to another proof of the theorem given by London [13]. FranklFüredi-Kalai used a combinatorial technique called shifting, while our proof is based on compression, a technique which was introduced by Macaulay [14] and used efficiently by Clements-Lindström [4].

This paper is organized as follows: In Section 2, we show that colored squarefree rings are Macaulay-Lex. In Section 3, we study the graded Betti numbers of lexplus- $Q$ ideals and disprove Conjecture 1.2(2). In Section 4, we study the graded Betti numbers of Borel ideals of $r$-colored squarefree rings. In Section 5, we discuss some related problems.

\section{The Macaulay-Lex property}

In studying Problem 1.1 and Conjecture 1.2, it is enough to consider monomial ideals since the initial ideal of a homogeneous-plus- $M$ ideal $I$ is a monomial ideal which contains $M$ and which has the same Hilbert function as $I$ (see e.g., [6, Chap. 15]). Thus, throughout this paper, we assume that all the ideals are monomial ideals. The main result of this section is the following.

Theorem 2.1 Let $R$ be an $r$-colored squarefree ring. For any monomial ideal $I$ of $R$, there exists a unique lex ideal $L$ of $R$ having the same Hilbert function as $I$. 
In the rest of this section, $R=S / Q$ stands for an $r$-colored squarefree ring as defined in Definition 1.3. For a subset $\mathcal{A} \subset[r]=\{1,2, \ldots, r\}$, write $\overline{\mathcal{A}}=[r] \backslash \mathcal{A}, V_{\mathcal{A}}=$ $\bigcup_{j \in \mathcal{A}} V_{j}, S_{\mathcal{A}}=K\left[V_{\mathcal{A}}\right], Q_{\mathcal{A}}=\sum_{j \in \mathcal{A}}\left(x_{j, 1}, \ldots, x_{j, n_{j}}\right)^{2} \subset S_{\mathcal{A}}$ and $R_{\mathcal{A}}=S_{\mathcal{A}} / Q_{\mathcal{A}}$. For any set $W$ of monomials of $R$, we write $W_{d}$ for the set of monomials in $W$ of degree $d$.

Definition 2.2 Let $W$ be a set of monomials of $R$ and $\mathcal{A} \subset[r]$. Then we may decompose $W$ as the disjoint union

$$
W=\biguplus_{f \in R_{\overline{\mathcal{A}}}} f W_{f}
$$

where $f$ ranges over the monomials in $R_{\overline{\mathcal{A}}}$ and each $W_{f}$ is a set of monomials in $R_{\mathcal{A}}$. We say that $W$ is $\mathcal{A}$-compressed if all the $W_{f}$ are lex-segments of $R_{\mathcal{A}}$. Moreover, we say that $W$ is compressed if $W$ is $\mathcal{A}$-compressed for all $\mathcal{A} \subset[r]$ with $|\mathcal{A}|=r-1$, where $|\mathcal{A}|$ is the cardinality of $\mathcal{A}$. Let $L_{f}=\biguplus_{d>0}\left(L_{f}\right)_{d}$ be the lex-segment set of monomials in $R_{\mathcal{A}}$ such that $\left|\left(W_{f}\right)_{d}\right|=\left|\left(L_{f}\right)_{d}\right|$ for all $d$. The set of monomials $X=$ $\biguplus_{f} f L_{f}$ is called the $\mathcal{A}$-compression of $W$.

Let $I$ be a monomial ideal of $R$ and $M$ the set of monomials in $I$. The $\mathcal{A}$ compression of $I$ is the $K$-vector space spanned by the $\mathcal{A}$-compression of $M$.

Notation 2.3 Let $W$ be a set of monomials in $R_{d}$. Define

$$
\operatorname{Shad}(W)=\left\{y m \in R_{d+1}: y \in V, m \in W\right\} .
$$

Write $\operatorname{Lex}(W) \subset R_{d}$ for the lex-segment set of monomials with $|W|=|\operatorname{Lex}(W)|$.

For any monomial $m \in R$, let first $(m)$ (resp. last $(m))$ be the greatest (resp. smallest) variable which divides $m$. Let

$$
\operatorname{color}(m)=\left\{j \in[r]: \text { there exists } y \in V_{j} \text { such that } y \text { divides } m\right\} \text {. }
$$

The following facts are straightforward (see, e.g., [17]).

Lemma 2.4 If $W \subset R$ is a lex-segment set of monomials of the same degree then $\operatorname{Shad}(W)$ is also a lex-segment.

Corollary 2.5 Theorem 2.1 holds if and only if, for any set $W \subset R$ of monomials of the same degree, one has $|\operatorname{Shad}(W)| \geq|\operatorname{Shad}(\operatorname{Lex}(W))|$.

We will prove Theorem 2.1 by using Corollary 2.5 and induction on $r$.

Lemma 2.6 Theorem 2.1 holds if $r \leq 2$.

Proof The statement is obvious if $r=1$. Suppose $r=2$. Note that $R=R_{0} \oplus R_{1} \oplus R_{2}$. Then, by Corollary 2.5, it is enough to show that, for any set $W \subset R_{1}$ of monomials, one has $|\operatorname{Shad}(W)| \geq|\operatorname{Shad}(\operatorname{Lex}(W))|$.

Let $a_{k}=\left|W \cap V_{k}\right|$ for $k=1,2$. A routine computation implies

$$
|\operatorname{Shad}(W)|=n_{1} a_{2}+n_{2} a_{1}-a_{1} a_{2}=-\left(n_{1}-a_{1}\right)\left(n_{2}-a_{2}\right)+n_{1} n_{2} .
$$


Then $|\operatorname{Shad}(W)|$ is smallest when the difference between $\left(n_{1}-a_{1}\right)$ and $\left(n_{2}-a_{2}\right)$ is minimized. Hence $|\operatorname{Shad}(W)| \geq|\operatorname{Shad}(\operatorname{Lex}(W))|$ by the definition of $>_{\text {lex }}$.

Definition 2.7 Let $W=\left\{u_{1}, \ldots, u_{t}\right\}$ and $W^{\prime}=\left\{u_{1}^{\prime}, \ldots, u_{t}^{\prime}\right\}$ be sets of monomials of $R$ with $u_{1}>_{\text {lex }} \cdots>_{\text {lex }} u_{t}$ and $u_{1}^{\prime}>_{\text {lex }} \cdots>_{\text {lex }} u_{t}^{\prime}$. We say that $W$ is lex-greater than $W^{\prime}$ if there exists $1 \leq j \leq t$ such that $u_{k}=u_{k}^{\prime}$ for $k<j$ and $u_{j}>_{\text {lex }} u_{j}^{\prime}$.

Lemma 2.8 Suppose that Theorem 2.1 holds for all $(r-1)$-colored squarefree rings. Let $\mathcal{A} \subset[r]$ with $|\mathcal{A}|=r-1$.

(i) For any monomial ideal I of $R$, the $\mathcal{A}$-compression of $I$ is an ideal of $R$.

(ii) Let $W \subset R_{d}$ be a set of monomials and $W^{\prime}$ the $\mathcal{A}$-compression of $W$. Then $W^{\prime}$ is lex-greater than or equal to $W$ and $|\operatorname{Shad}(W)| \geq\left|\operatorname{Shad}\left(W^{\prime}\right)\right|$.

Proof (i) Let $M=\bigoplus_{f} f M_{f}$ be the set of monomials in $I$, where $f \in R_{\overline{\mathcal{A}}}$ is a monomial and $M_{f} \subset R_{\mathcal{A}}$. Let $L=\bigoplus_{f} f L_{f}$ be the $\mathcal{A}$-compression of $M$. Note that the vector space spanned by $M_{f}$ is a monomial ideal of $R_{\mathcal{A}}$. By the assumption, the vector space spanned by $L_{f}$ is an ideal of $R_{\mathcal{A}}$. Hence, what we must prove is that, for any $f u \in f L_{f}$ and for any variable $y \in V_{\overline{\mathcal{A}}}$, one has $y f u \in L$ or $y f u=0$.

Suppose $y f \neq 0$. Since $I$ is an ideal, $W_{f} \subset W_{y f}$. Hence $L_{f} \subset L_{y f}$. This implies $u \in L_{y f}$ and $y f u \in y f L_{y f} \subset L$.

(ii) It is clear that $W^{\prime}$ is lex-greater than or equal to $W$. Let $I$ be the monomial ideal generated by $W$ and $J$ the $\mathcal{A}$-compression of $I$. Then $|\operatorname{Shad}(W)|=\operatorname{dim}_{K} I_{d+1}$ and $\left|\operatorname{Shad}\left(W^{\prime}\right)\right| \leq \operatorname{dim}_{K} J_{d+1}=\operatorname{dim}_{K} I_{d+1}$ by (i). Hence the statement follows.

Lemma 2.9 Let $W \subset R_{d}$ be a compressed set of monomials. Let $u \in W$ and $v \in R_{d}$. If $v>_{\operatorname{lex}} u$ and if $u$ and $v$ are divisible by some variable $y$ then $v \in W$.

Proof Let $\mathcal{A}=[r] \backslash \operatorname{color}(y)$. Since $W$ is $\mathcal{A}$-compressed and $\frac{u}{y} \in W_{y}$, we have $\frac{v}{y} \in$ $W_{y}$, i.e., $v \in W$.

For any monomial $m \in R$, write

$$
\operatorname{grow}(m)=\left|\left\{y \in V_{[r] \backslash \operatorname{color}(m)}: y<\operatorname{last}(m)\right\}\right| .
$$

Note that if $W \subset R_{d}$ is a lex-segment, then $|\operatorname{Shad}(W)|=\sum_{m \in W} \operatorname{grow}(m)$. This definition is inspired by work of Bigatti [2], who used the analogous formula in a polynomial ring to study Borel ideals.

Lemma 2.10 Let $m$ and $m^{\prime}$ be monomials of degree $d$ with $\operatorname{last}(m)=x_{s, t}$ and $\operatorname{last}\left(m^{\prime}\right)=x_{s^{\prime}, t^{\prime}}$. If $t<t^{\prime}$ then $\operatorname{grow}(m) \leq \operatorname{grow}\left(m^{\prime}\right)$.

Proof We may assume $t^{\prime}=t+1$. For any $j \in \operatorname{color}(m)$ one has

$$
\left|\left\{y \in V_{j}: y \leq x_{1, t}\right\}\right|=t .
$$

Indeed, if $\left|\left\{y \in V_{j}: y \leq x_{1, t}\right\}\right| \leq t-1$ then $n_{j} \leq t-1$. However, since $j \in$ color $(m)$, some $x_{j, \ell}$ is greater than $x_{s, t}$. This means $n_{j} \geq \ell \geq t$, a contradiction. Then (1) implies grow $\left(m^{\prime}\right) \geq\left|\left\{y \in V: y \leq x_{1, t}\right\}\right|-d t \geq \operatorname{grow}(m)$. 
Lemma 2.11 Suppose $r \geq 3$. Let $W \subset R_{d}$ be a compressed set of monomials which is not a lex-segment. There exists a set $W^{\prime} \subset R_{d}$ of monomials such that $|W|=\left|W^{\prime}\right|$, $|\operatorname{Shad}(W)| \geq\left|\operatorname{Shad}\left(W^{\prime}\right)\right|$, and $W^{\prime}$ is lex-greater than $W$.

Proof If $r \geq 3$ then any compressed subset $W \subset R_{1}$ is a lex-segment. Suppose $d \geq 2$. Let $g \in R_{d}$ be the lex-greatest monomial which is not in $W$ and $b$ the lex-smallest monomial in $W$. Set $\tilde{W}=W \cup\{g\}$ and $W^{\prime}=(W \backslash\{b\}) \cup\{g\}$. Then, by the choice of $g$ and $b$, a straightforward computation implies

$$
|\operatorname{Shad}(\tilde{W})| \leq|\operatorname{Shad}(W)|+\operatorname{grow}(g) \quad \text { and } \quad|\operatorname{Shad}(\tilde{W})| \geq\left|\operatorname{Shad}\left(W^{\prime}\right)\right|+\operatorname{grow}(b) \text {. }
$$

Hence, to prove the statement, it is enough to show

$$
\operatorname{grow}(g) \leq \operatorname{grow}(b)
$$

The statement is obvious if $d=r$. Hence we may assume $2 \leq d<r$. Let $u$ be the lex-greatest monomial in $W$ such that $u<_{\text {lex }} g$. Set $y_{1}=\operatorname{first}(u), y_{0}=\operatorname{first}(g)$ and $z=\operatorname{last}(b)$. Clearly, $y_{0} \geq y_{1}$ since $g>_{\text {lex }} u$. Moreover, since $W$ is compressed, Lemma 2.9 implies

$$
y_{0}>y_{1} \text {. }
$$

[Case 1]: Suppose $\operatorname{color}\left(y_{0}\right) \neq \operatorname{color}(z)$. Since $u \geq_{\text {lex }} b$, we have $y_{0}>y_{1} \geq$ first $(b)$. Let $f$ be the lex-smallest monomial of degree $d$ which is divisible by $y_{0} z$. Since $y_{0}>\operatorname{first}(b), f>_{\text {lex }} b$. Since $f$ and $b$ are divisible by $z$, Lemma 2.9 implies $f \in W$. Also, since $f>_{\operatorname{lex}} u$ and since $u$ is the lex-greatest monomial in $W$ with $u<$ lex $g$, we have $f>_{\text {lex }} g$.

Let $w=\operatorname{first}\left(\frac{f}{y_{0}}\right)$. Since $f$ is the lex-smallest monomial of degree $d$ which is divisible by $y_{0} z$, if $w \neq z$ then $f$ is the lex-smallest monomial of degree $d$ which is divisible by $y_{0}$. However, this cannot happen since $f>_{\operatorname{lex}} g$ and $g$ is divisible by $y_{0}$. Hence $z=w$. Write $g=y_{0} w_{1} \cdots w_{d-1}$, where $y_{0}>w_{1}>\cdots>w_{d-1}$. Since first $\left(\frac{f}{y_{0}}\right)=z, \frac{f}{y_{0}}>_{\text {lex }} \frac{g}{y_{0}}$, and $\frac{f}{y_{0}}$ is the lex-smallest monomial of degree $d-1$ which is divisible by $z$, we have $z>w_{1}>\cdots>w_{d-1}$. Let $z=x_{p, q}$ and $w_{d-1}=x_{s, t}$. Note that $t \leq q$ since $z>w_{d-1}$. If $t<q$ then, by Lemma 2.10, we have $\operatorname{grow}(g) \leq \operatorname{grow}(b)$ as desired. If $t=q$ then, for each $k, w_{k}=x_{\ell_{k}, q}$ for some $p<\ell_{k} \leq s$. Hence, by (1),

$$
\begin{aligned}
\operatorname{grow}(b) & \geq\left|\left\{y \in V_{[r] \backslash \operatorname{color}\left(y_{0}\right)}: y<x_{p, q}\right\}\right|-(d-1) q \\
& \geq\left|\left\{y \in V_{[r] \backslash \operatorname{color}\left(y_{0}\right)}: y<x_{s, t}\right\}\right|+(d-1)-(d-1) q \\
& =\left|\left\{y \in V_{[r] \backslash \operatorname{color}\left(y_{0}\right)}: y<x_{s, t}\right\}\right|-(d-1)(q-1)=\operatorname{grow}(g) .
\end{aligned}
$$

[Case 2]: Suppose $\operatorname{color}\left(y_{0}\right)=\operatorname{color}(z)=\{c\}$. Fix $a \in[r] \backslash \operatorname{color}(b)$. Note that $\{a\} \neq \operatorname{color}\left(y_{0}\right)$. Let $f=y_{0} f^{\prime}$ be the lex-smallest monomial of degree $d$ such that $f$ is divisible by $y_{0}$ and $a \notin \operatorname{color}(f)$. Since $y_{0}>y_{1}, f>_{\text {lex }} u \geq_{\text {lex }} b$. Then, since $f, b \in R_{[r] \backslash\{a\}}$ and $W$ is compressed, we have $f \in W$. In particular, $f>_{\text {lex }} g$ by the choice of $u$. Hence $f^{\prime}$ cannot be the lex-smallest monomial of degree $d-1$ in $R_{[r] \backslash\{c\}}$. 
Let $m$ be the lex-smallest monomial of degree $d$ in $R_{[r] \backslash\{c\}}$. Since $f^{\prime}$ is lex-smallest in $\left(R_{[r] \backslash\{a, c\}}\right)_{d-1}$ but is not lex-smallest in $\left(R_{[r] \backslash\{c\}}\right)_{d-1}, m$ is divisible by $x_{a, 1}$ and $f^{\prime}=\frac{m}{x_{a, 1}}$. Then $\frac{g}{y_{0}}<_{\operatorname{lex}} f^{\prime} \leq_{\operatorname{lex}} \frac{m}{\operatorname{last}(m)}$. Since $m$ is lex-smallest in $\left(R_{[r] \backslash\{c\}}\right)_{d}$,

$$
\operatorname{last}(g)=\operatorname{last}(m)= \begin{cases}x_{r, 1}, & \text { if } c \neq r \\ x_{r-1,1}, & \text { if } c=r\end{cases}
$$

In both cases, we have $\operatorname{grow}(g)=0 \leq \operatorname{grow}(b)$.

Now we are in position to prove Theorem 2.1.

Proof of Theorem 2.1 We use induction on $r$. We may assume $r \geq 3$ by Lemma 2.6. Suppose that the statement holds for all $(r-1)$-colored squarefree rings.

Let $W$ be a set of monomials of degree $d$. By Corollary 2.5, it is enough to show that $|\operatorname{Shad}(W)| \geq|\operatorname{Shad}(\operatorname{Lex}(W))|$. By Lemmas 2.8 and 2.11, if $W$ is not a lex-segment then there exists $W^{\prime} \subset R_{d}$ such that $\left|W^{\prime}\right|=|W|,\left|\operatorname{Shad}\left(W^{\prime}\right)\right| \leq$ $|\operatorname{Shad}(W)|$ and $W^{\prime}$ is lex-greater than $W$. Arguing inductively, we have $|\operatorname{Shad}(W)| \geq$ $|\operatorname{Shad}(\operatorname{Lex}(W))|$.

Remark 2.12 Here we note the relation between Theorem 2.1 and face vectors of colored simplicial complexes. A simplicial complex $\Delta$ on $[n]=\{1,2, \ldots, n\}$ is a collection of subsets of $[n]$ such that, if $F \in \Delta$ and $G \subset F$, then $G \in \Delta$. A simplicial complex $\Delta$ is said to be $r$-colored if there exists a partition of $[n],[n]=C_{1} \cup \cdots \cup C_{r}$, such that for every $F \in \Delta$ and every $1 \leq i \leq r,\left|C_{i} \cap F\right| \leq 1$. In particular, if $r=$ $\max \{|F|: F \in \Delta\}$ then $\Delta$ is called completely balanced.

Let $H_{i}=\{k \in[n]: k \equiv i(\bmod r)\}$ for $i=1,2, \ldots, r$ and let $\mathcal{C}$ be the collection of subsets $F \subset[n]$ satisfying $\left|F \cap H_{i}\right| \leq 1$ for all $i$. Let $>_{\text {rev }}$ be the reverse lexicographic order induced by $1>_{\text {rev }} \cdots>_{\text {rev }} n$. An $r$-colored rev-lex complex $\Gamma \subset \mathcal{C}$ is an $r$ colored simplicial complex such that, for any faces $F \in \Gamma$ and $G \in \mathcal{C}$ with $|F|=$ $|G|$, if $G>_{\text {rev }} F$ then $G \in \Gamma$. Considering the partition $[n]=H_{1} \cup \cdots \cup H_{r}$, we see that $r$-colored rev-lex complexes are $r$-colored. While many $r$-colored complexes are not defined by the special partition above, Frankl, Füredi and Kalai [8] proved that they all share a face vector with an $r$-colored rev-lex complex. In particular, since $r$-colored rev-lex complexes are uniquely determined by their face vectors, this result characterizes the possible face vectors of colored complexes in terms of colored revlex complexes (a numerical characterization was also given in [8]). This result of Frankl, Füredi and Kalai can be recovered from Theorem 2.1.

Suppose that $\Delta$ is an $r$-colored simplicial complex on $[n]$. Then there exists a monomial ideal $I$ of an $r$-colored squarefree ring $R$ of type $(n, \ldots, n)$ such that the set of monomials of $R$ which are not in $I$ can be identified with $\Delta$. (Since $R$ has $n r$ variables, the ideal $I$ will contain at least $(n r-n)$ variables corresponding to vertices which do not appear in $\Delta$.) Theorem 2.1 shows that there exists a lex ideal $L$ of $R$ having the same Hilbert function as $I$. The $r$-colored rev-lex complex having the same face vector as $\Delta$ corresponds to the set of monomials of $R$ which are not in $L$.

Actually, Theorem 2.1 refines this result. Theorem 2.1 characterizes the face vectors of colored simplicial complexes on $[n]$ with a fixed partition $[n]=C_{1} \cup \cdots \cup C_{r}$. 
For example, our result gives the complete description of face vectors of 2-colored complexes on $\{1,2,3,4,5,6\}$ with the specific partition $\{1,2,3,4\} \cup\{5,6\}$, while the Frankl-Füredi-Kalai theorem does not guarantee this.

\section{Betti numbers of lex-plus- $Q$ ideals}

In this section, we show that most colored squarefree rings do not admit ideals with maximal Betti numbers over $S$. As before, let $R=S / Q$ be an $r$-colored squarefree ring of type $\left(n_{1}, \ldots, n_{r}\right)$ as defined in Definition 1.3. For a finitely generated graded $S$-module $M$, the integers $\beta_{i, j}(M)=\operatorname{dim}_{K} \operatorname{Tor}_{i}(M, K)_{j}$ and $\beta_{i}(M)=$ $\operatorname{dim}_{K} \operatorname{Tor}_{i}(M, K)$ are called the graded Betti numbers of $M$ and the total Betti numbers of $M$ respectively. A monomial ideal $I$ of $S$ is said to be strongly color-stable if $u x_{j, k} \in I$ implies $u x_{j, \ell} \in I$ for all $k<\ell$ and for all $j \in[r]$. The next fact easily follows from [1, Theorem 5.4] or [15, Theorem 5.9].

Lemma 3.1 Let I be a homogeneous-plus- $Q$ ideal. There exists a strongly colorstable ideal $J$ with $J \supset Q$ and $\operatorname{Hilb}(J)=\operatorname{Hilb}(I)$ such that $\beta_{i j}(J) \geq \beta_{i j}(I)$ for all $i$ and $j$.

The above lemma shows that, to study Conjecture 1.2(2) for colored squarefree rings, it is enough to consider strongly color-stable ideals.

\subsection{2-colored squarefree rings}

We first consider 2 -colored squarefree rings. Let $n_{1} \geq n_{2} \geq 4, S=K\left[x_{1}, \ldots, x_{n_{1}}\right.$, $\left.y_{1}, \ldots, y_{n_{2}}\right]$ and $Q=\left(x_{1}, \ldots, x_{n_{1}}\right)^{2}+\left(y_{1}, \ldots, y_{n_{2}}\right)^{2}$.

Proposition 3.2 A 2-colored squarefree ring $R=S / Q$ of type $\left(n_{1}, n_{2}\right)$ with $n_{2} \geq 4$ does not admit ideals with maximal Betti numbers over $S$.

We first give an example.

Example 3.3 Let $A=K\left[x_{1}, \ldots, x_{4}, y_{1}, \ldots, y_{4}\right]$ and $P=\left(x_{1}, \ldots, x_{4}\right)^{2}+\left(y_{1}, \ldots, y_{4}\right)^{2}$. Let

$$
\begin{gathered}
L=\left(x_{4}, x_{3}, y_{4}, x_{2} y_{3}, x_{1} y_{3}\right)+P, \\
I=\left(x_{4}, x_{3}, y_{4}, x_{2} y_{3}, x_{2} y_{2}\right)+P
\end{gathered}
$$

and

$$
J=\left(x_{4}, x_{3}, x_{2}\right)+P .
$$

Then $L$ is lex-plus- $P$ and any strongly color-stable ideal $B$ with $B \supset P$ and $\operatorname{Hilb}(B)=\operatorname{Hilb}(L)$ is isomorphic to $L, I$, or $J$. The following are Betti diagrams of these ideals computed by the computer algebra system Macaulay 2 [9]: 


\begin{tabular}{|c|c|c|c|c|c|c|c|c|c|}
\hline etti (res (S/L)) & $=$ total & $\perp$ & 14 & 65 & 156 & 224 & 202 & 113 & 36 \\
\hline & 0 : & 1 & 3 & 3 & 1 & • & . & . & . \\
\hline & $1:$ & . & 11 & 53 & 107 & 118 & 78 & 32 & 8 \\
\hline & $2:$ & . & . & 9 & 48 & 106 & 124 & 81 & 28 \\
\hline etti (res (S/I)) & $=$ total: & 1 & 14 & 66 & 159 & 225 & 196 & 104 & 31 \\
\hline & $0:$ & 1 & 3 & 3 & 1 & . & . & . & . \\
\hline & $1:$ & . & 11 & 53 & 108 & 120 & 77 & 27 & 4 \\
\hline & $2:$ & . & . & 10 & 50 & 105 & 119 & 77 & 27 \\
\hline betti (res (S/J)) & $=$ total: & 1 & 14 & 64 & 150 & 209 & 182 & 98 & 304 \\
\hline & 0 & 1 & 3 & 3 & 1 & . & & . & . \\
\hline & & & 11 & 53 & 106 & 113 & 68 & 22 & 3. \\
\hline & & & . & 8 & 43 & 96 & 114 & 76 & $27 \quad 4$ \\
\hline
\end{tabular}

None of these ideals has maximal graded Betti numbers. Hence, by Lemma 3.1, the ring $A / P$ does not admit ideals with maximal Betti numbers.

Proof of Proposition 3.2 We use the ideals given in Example 3.3. Let $L^{\prime}$ be the ideal of $S$ defined by

$$
L^{\prime}=L S+\left(x_{5}, \ldots, x_{n_{1}}, y_{5}, \ldots, y_{n_{2}}\right) .
$$

Define $I^{\prime}$ and $J^{\prime}$ in the same way as $L^{\prime}$. Then $L^{\prime}$ is lex-plus- $Q$, and every strongly color-stable ideal $B$ with $B \supset Q$ and $\operatorname{Hilb}(B)=\operatorname{Hilb}\left(L^{\prime}\right)$ is isomorphic to $L^{\prime}, I^{\prime}$, or $J^{\prime}$. Recall that, if $M$ is a homogeneous ideal of $S$ and $f$ is a non-zero divisor of $S / M$, then the tensor product of the minimal free resolutions of $S / M$ and $S /(f)$ is a minimal free resolution of $S /(M+(f))$. By using this fact together with the computations given in Example 3.3, it follows that

$$
\beta_{1}\left(I^{\prime}\right)>\beta_{1}\left(L^{\prime}\right)>\beta_{1}\left(J^{\prime}\right)
$$

and

$$
\beta_{n_{1}+n_{2}-1}\left(L^{\prime}\right)>\beta_{n_{1}+n_{2}-1}\left(I^{\prime}\right)=\beta_{n_{1}+n_{2}-1}\left(J^{\prime}\right) .
$$

Then, by Lemma 3.1, the ring $S / Q$ does not admit ideals with maximal Betti numbers over $S$.

Remark 3.4 While we used a computer system for the computations of Betti diagrams of $L, I$, and $J$, one can compute those Betti numbers by using Lemma 3.7 and Hochster's formula.

\subsection{General construction}

In the rest of this section, $R=S / Q$ is an $r$-colored squarefree ring of type $\left(n_{1}, \ldots, n_{r}\right)$ with $r \geq 3$ and $n_{r} \geq 3$. The goal is to show the following.

Theorem 3.5 With the same notation as above, $R$ does not admit ideals with maximal Betti numbers over $S$. 
First, we give a formula to compute the graded Betti numbers of a strongly colorstable ideal $I$ with $I \supset Q$ using simplicial complexes. Let $\tilde{V}_{j}=\left\{x_{j, 0}\right\} \cup V_{j}, \tilde{V}=$ $\bigcup_{j=1}^{r} \tilde{V}_{j}$ and $\tilde{S}=K[\tilde{V}]$. Let

$$
\tilde{Q}=\left(\left\{x_{j, k} x_{j, \ell} \in \tilde{S}: k \neq \ell, j=1,2, \ldots, r\right\}\right)
$$

For any strongly color-stable ideal $I=I^{\prime}+Q$ of $S$, where $I^{\prime}$ is generated by monomials not in $Q$, define

$$
\tilde{I}=I^{\prime} \tilde{S}+\tilde{Q} .
$$

Thus the ideal $\tilde{I}$ is obtained from $I=I^{\prime}+Q$ by replacing $Q$ by $\tilde{Q}$.

Example 3.6 Let $I=\left(x_{1,2} x_{2,2}, x_{1,2} x_{2,1}\right)+\left(x_{1,1}^{2}, x_{1,1} x_{1,2}, x_{1,2}^{2}, x_{2,1}^{2}, x_{2,1} x_{2,2}, x_{2,2}^{2}\right)$. Then

$$
\tilde{I}=\left(x_{1,2} x_{2,2}, x_{1,2} x_{2,1}\right)+\left(x_{1,0} x_{1,1}, x_{1,0} x_{1,2}, x_{1,1} x_{1,2}, x_{2,0} x_{2,1}, x_{2,0} x_{2,2}, x_{2,1} x_{2,2}\right) .
$$

Actually, the map $I \rightarrow \tilde{I}$ is a special case of the colored squarefree operation introduced in [1]. Thus, by [21, Theorem 0.1 ], we obtain

Lemma 3.7 Let I be a strongly color-stable ideal of $S$ with $I \supset Q$. Then I and $\tilde{I}$ have the same graded Betti numbers.

Since $\tilde{I}$ is a squarefree monomial ideal, we can compute its graded Betti numbers using Hochster's formula. We recall Hochster's formula. Let $J$ be a squarefree monomial ideal of $\tilde{S}$, and let $\Delta(J)$ be its Stanley-Reisner complex,

$$
\Delta(J)=\{u \in \tilde{S} \backslash J: u \text { is a squarefree monomial }\} .
$$

(We identify squarefree monomials of $\tilde{S}$ with subsets of $\tilde{V}$ and regard $\Delta(J)$ as a simplicial complex on the vertex set $\tilde{V}$.) Hochster's formula [11] says

$$
\beta_{i, j}(J)=\sum_{W \subset \tilde{V},|W|=j} \operatorname{dim}_{K} \tilde{H}_{j-i-2}\left(\Delta(J)_{W} ; K\right),
$$

where $\Delta(J)_{W}=\Delta(J) \cap K[W]$ and where $\tilde{H}_{i}(\Delta ; K)$ is the $i$-th reduced homology group of a simplicial complex $\Delta$ over a field $K$.

A simplicial complex $\Delta$ on the vertex set $\tilde{V}$ is said to be colored shifted if $u x_{j, k} \in \Delta$ implies $u x_{j, \ell} \in \Delta$ for any $j \in[r]$ and for any $0 \leq \ell<k$ such that $x_{j, \ell}$ does not divide $u$. The top Betti numbers of colored shifted complexes can be computed as follows (see [1, Theorem 5.7] and [21, Proposition 4.2]).

Lemma 3.8 (Babson-Novik) If $\Delta$ is a colored shifted simplicial complex on $\tilde{V}$ then $\operatorname{dim}_{K} \tilde{H}_{r-1}(\Delta ; K)=\mid\left\{u \in \Delta: \operatorname{deg} u=r, u\right.$ is not divisible by any of $\left.x_{j, 0}\right\} \mid$. 
Let $I$ be a strongly color-stable ideal of $S$. Define

$$
\Gamma(I)=\{u \in S \backslash I: u \text { is a squarefree monomial with } \operatorname{deg} u=r\} .
$$

Lemma 3.9 If I is a strongly color-stable ideal of $S$ with I $\supset Q$, then

$$
\beta_{r-1,2 r}(I)=\sum_{x_{1, i_{1}} x_{2, i_{2}} \cdots x_{r, i_{r}} \in \Gamma(I)}\left(\prod_{k=1}^{r} i_{k}\right) .
$$

Proof Note that $\Gamma(I)$ is the set of monomials $u$ in $\Delta(\tilde{I})$ of degree $r$ which are not divisible by any of the $x_{j, 0}$. Since $I$ is strongly color-stable, the simplicial complex $\Delta(\tilde{I})$ is colored shifted. Note also that, for any $W \subset \tilde{V}, \Delta(\tilde{I})_{W}$ is colored shifted on $W$. Hence, for any $W \subset \tilde{V}$ with $|W|=2 r$, Lemma 3.8 implies

$$
\operatorname{dim}_{K} \tilde{H}_{r-1}\left(\Delta(\tilde{I})_{W} ; K\right)= \begin{cases}1, & \text { if }\left|W \cap V_{j}\right|=2 \text { for all } j \text { and } \Delta(\tilde{I})_{W} \text { contains all } \\ \text { monomials of the form } x_{1, i_{1}} \cdots x_{r, i_{r}} \in K[W] & \\ 0, & \text { otherwise. }\end{cases}
$$

(Observe that, if $W \cap V_{j}=\left\{x_{j, k}, x_{j, \ell}\right\}$ with $k<l$, then $x_{j, k}$ plays the role of $x_{j, 0}$ in Lemma 3.8.)

By Hochster's formula,

$$
\begin{aligned}
\beta_{r-1,2 r}(\tilde{I}) & =\sum_{W \subset \tilde{V},|W|=2 r} \operatorname{dim}_{K} \tilde{H}_{r-1}\left(\Delta(\tilde{I})_{W} ; K\right) \\
& =\left|\left\{W=\bigcup_{j=1}^{r}\left\{x_{j, p_{j}}, x_{j, q_{j}}\right\} \subset \tilde{V}: p_{j}<q_{j}, \prod_{j=1}^{r} x_{j, q_{j}} \in \Delta(\tilde{I})\right\}\right| \\
& =\sum_{x_{1, q_{1}} x_{2, q_{2}} \cdots x_{r, q_{r}} \in \Gamma(I)}\left(\prod_{k=1}^{r} q_{k}\right) .
\end{aligned}
$$

The last equality follows since $I$ is strongly color-stable. Then the statement follows from Lemma 3.7.

We need the following fact.

Lemma 3.10 Let $a_{1} \geq \cdots \geq a_{t}$ and $b_{1} \geq \cdots \geq b_{t}$ be two sequences of integers. If $a_{k}+\cdots+a_{t} \geq b_{k}+\cdots+b_{t}$ for all $k$ then $a_{1} \cdots a_{t} \geq b_{1} \cdots b_{t}$.

Proof We induct on $t$. If $t=1$ then there is nothing to prove. Suppose $t>1$. If $a_{1} \geq b_{1}$ then the statement immediately follows by induction. Suppose $a_{1}<b_{1}$. Note that $a_{2}+\cdots+a_{t}>b_{2}+\cdots+b_{t}$ by the assumption. Let $p \geq 2$ be the greatest integer such that $a_{p}=a_{2}$. We claim that $a_{k}+\cdots+a_{t}>b_{k}+\cdots+b_{t}$ for $2 \leq k \leq p$.

Suppose to the contrary that $a_{k}+\cdots+a_{t}=b_{k}+\cdots+b_{t}$ for some $k, 2<k \leq p$. Since $a_{k+1}+\cdots+a_{t} \geq b_{k+1}+\cdots+b_{t}$, we have

$$
a_{2}=\cdots=a_{k} \leq b_{k} \leq \cdots \leq b_{2} .
$$

Then $\sum_{j=2}^{t} a_{j}=\sum_{j=2}^{k-1} a_{j}+\sum_{j=k}^{t} a_{j} \leq \sum_{j=2}^{t} b_{j}$, a contradiction. 
Thus, the sequence $\left(a_{1}+1, a_{2}, \ldots, a_{p-1}, a_{p}-1, a_{p+1}, \ldots, a_{t}\right)$ also satisfies the assumption of the lemma, and, since $a_{1} \geq a_{p}$,

$$
a_{1} a_{p}\left(\prod_{j \neq 1, p} a_{j}\right) \geq\left(a_{1}+1\right)\left(a_{p}-1\right)\left(\prod_{j \neq 1, p} a_{j}\right) \text {. }
$$

By repeating this procedure, we may assume $a_{1}=b_{1}$. The statement follows by induction.

Proof of Theorem 3.5 Let

$$
\alpha=x_{1, n_{1}} x_{2, n_{2}} \cdots x_{r-2, n_{r-2}} .
$$

[Case 1]: Suppose $n_{r-1}=n_{r}$. To simplify, set $n=n_{r-1}=n_{r}$. Let

$$
I=\left(\alpha x_{r-1, n}, \alpha x_{r-1, n-1}\right)+Q
$$

and

$$
L=\left(\alpha x_{r-1, n}, \alpha x_{r, n}, \alpha x_{r-1, n-1} x_{r, n-1}\right)+Q .
$$

Note that $I$ and $L$ are strongly color-stable ideals having the same Hilbert function. First, we show that the graded Betti numbers of $I$ and $L$ are incomparable.

Clearly, $\beta_{0}(I)<\beta_{0}(L)$. It is enough to show that $\beta_{r-1,2 r}(I)>\beta_{r-1,2 r}(L)$. Since

$$
\Gamma(I) \backslash \Gamma(L)=\left\{\alpha x_{r, n} x_{r-1, \ell}: \ell=1,2, \ldots, n-2\right\}
$$

and

$$
\Gamma(L) \backslash \Gamma(I)=\left\{\alpha x_{r-1, n-1} x_{r, \ell}: \ell=1,2, \ldots, n-2\right\},
$$

it follows from Lemma 3.9 that

$$
\begin{aligned}
& \beta_{r-1,2 r}(I)-\beta_{r-1,2 r}(L) \\
& =\sum_{x_{1, i_{1}} x_{2, i_{2}} \cdots x_{r, i_{r}} \in \Gamma(I) \backslash \Gamma(L)}\left(\prod_{k=1}^{r} i_{k}\right)-\sum_{x_{1, i_{1}} x_{2, i_{2}} \cdots x_{r, i_{r}} \in \Gamma(L) \backslash \Gamma(I)}\left(\prod_{k=1}^{r} i_{k}\right) \\
& =\left(\prod_{j=1}^{r-2} n_{j}\right)\{n-(n-1)\}(1+2+\cdots+(n-2))>0 .
\end{aligned}
$$

Since $L$ is lex-plus- $Q$, any monomial ideal $J \supset Q$ with $\operatorname{Hilb}(J)=\operatorname{Hilb}(L)$ satisfies $\beta_{0}(J) \leq \beta_{0}(L)$. Thus, to complete the proof, it is enough to show that, if $J$ is any homogeneous-plus- $Q$ ideal which has the same Hilbert function as $L$ and satisfies $\beta_{0}(J)=\beta_{0}(L)$, then $\beta_{r-1,2 r}(J) \leq \beta_{r-1,2 r}(L)$.

We may assume that $J$ is strongly color-stable. Since $J$ has the same Hilbert function as $L, J$ contains two monomials $u_{1}$ and $u_{2}$ of degree $r-1$ which are not in $Q$. Let $\left\{c_{k}\right\}=[r] \backslash \operatorname{color}\left(u_{k}\right)$. Then $\left|\operatorname{Shad}\left(\left\{u_{1}, u_{2}\right\}\right)\right| \geq n_{c_{1}}+n_{c_{2}}-1$. Since $J$ has a generator 
of degree $r$ and has the same Hilbert function as $L,\left|\operatorname{Shad}\left(\left\{u_{1}, u_{2}\right\}\right)\right|$ must be equal to $2 n-1$. It follows that $n_{c_{1}}=n_{c_{2}}=n$, and $c_{1} \neq c_{2}$. Thus $\left(u_{1}, u_{2}\right)+Q$ is isomorphic to $\left(\alpha x_{r-1, n}, \alpha x_{r, n}\right)+Q$. Hence we may assume $u_{1}=\alpha x_{r-1, n}$ and $u_{2}=\alpha x_{r, n}$.

Let $v$ be a generator of $J$ of degree $r$. Then $\Gamma(J) \backslash \Gamma(L)=\left\{\alpha x_{r-1, n-1} x_{r, n-1}\right\}$ and $\Gamma(L) \backslash \Gamma(J)=\{v\}$. Also, since $J \neq L$ is strongly color-stable, $v$ is a monomial of the form $v=x_{k, n_{k}-1}\left(\prod_{j \neq k} x_{j, n_{j}}\right)$ for some $1 \leq k \leq r-2$. Then, by Lemma 3.9, we have

$$
\beta_{r-1,2 r}(L)-\beta_{r-1,2 r}(J) \geq\left(\prod_{j \neq k, r-1, r} n_{j}\right)\left\{\left(n_{k}-1\right) n^{2}-n_{k}(n-1)^{2}\right\} \geq 0
$$

as desired.

[Case 2]: Suppose $n_{r-1}>n_{r}$. Let

$$
I=\alpha\left(x_{r-1, n_{r-1}}, \ldots, x_{r-1, n_{r}+2}, x_{r, n_{r}}\right)+Q
$$

and

$$
L=\alpha\left(x_{r-1, n_{r-1}}, \ldots, x_{r-1, n_{r}+2}, x_{r-1, n_{r}+1}, x_{r-1, n_{r}} x_{r, n_{r}}\right)+Q .
$$

Then $I$ and $L$ are strongly color-stable ideals having the same Hilbert function. First, we show that the graded Betti numbers of $I$ and those of $L$ are incomparable. Clearly, $\beta_{0}(I)<\beta_{0}(L)$. On the other hand, in the same way as in [Case 1], we have $\beta_{r-1,2 r}(I)>\beta_{r-1,2 r}(L)$ since

$$
\Gamma(I) \backslash \Gamma(L)=\left\{\alpha x_{r-1, n_{r}+1} x_{r, \ell}: \ell=1,2, \ldots, n_{r}-1\right\}
$$

and

$$
\Gamma(L) \backslash \Gamma(I)=\left\{\alpha x_{r, n_{r}} x_{r-1, \ell}: \ell=1,2, \ldots, n_{r}-1\right\} .
$$

Since $L$ is lex-plus- $Q$, to complete the proof, it is enough to show that, for any strongly color-stable ideal $J \supset Q$ having the same Hilbert function as $L$, if $\beta_{0}(J)=$ $\beta_{0}(L)$ then $\beta_{r-1,2 r}(J) \leq \beta_{r-1,2 r}(L)$.

Let $t=n_{r-1}-n_{r}$. Then $J$ has generators $u_{1}, \ldots, u_{t}$ of degree $r-1$ which are not in $Q$. First, we claim that color $\left(u_{k}\right)=[r-1]$ for all $k$.

Let $\left\{c_{k}\right\}=[r] \backslash \operatorname{color}\left(u_{k}\right)$. Suppose $c_{1}=\cdots=c_{s}=r$ and $c_{k} \neq r$ for $k>s$. Then

$$
\left|\operatorname{Shad}\left(\left\{u_{1}, \ldots, u_{t}\right\}\right)\right| \geq s n_{r}+\left(n_{c_{s+1}}-s\right)+\left(n_{c_{s+2}}-s-1\right)+\cdots+\left(n_{c_{t}}-t+1\right) .
$$

However, since $J$ has a generator of degree $r$ and has the same Hilbert function as $L$, $\left|\operatorname{Shad}\left(\left\{u_{1}, \ldots, u_{t}\right\}\right)\right|$ must be equal to $t n_{r}$. Since $n_{c_{k}}-(k-1) \geq n_{r-1}-t+1>n_{r}$ for $k=s+1, \ldots, t$, it follows that $s=t$ and $\operatorname{color}\left(u_{k}\right)=[r-1]$ for all $k$.

Let $v=x_{1, j_{1}} \cdots x_{r, j_{r}}$ be the generator of $J$ with $\operatorname{deg} v=r$. Write

$$
u_{k}=x_{1, i_{1, k}} \cdots x_{r-1, i_{r-1, k}} .
$$

Since $J$ is strongly color-stable, we may assume that

$$
i_{1, k}+\cdots+i_{r-1, k} \geq n_{1}+\cdots+n_{r-1}-(k-1) \quad \text { for } k=1,2, \ldots, t
$$


and

$$
j_{1}+\cdots+j_{r-1} \geq n_{1}+\cdots+n_{r-1}-t .
$$

Also, since $J$ is strongly color-stable, we have

$$
j_{r}=n_{r} .
$$

Now, by Lemma 3.9, we have

$$
\begin{aligned}
\beta_{r-1,2 r}(L)-\beta_{r-1,2 r}(J) & \left.\sum_{x_{1, k_{1}} \cdots x_{r, k_{r}} \in \Gamma(L)} k_{1} \cdots k_{r}\right]-\left[\sum_{x_{1, k_{1}} \cdots x_{r, k_{r}} \in \Gamma(J)} k_{1} \cdots k_{r}\right] \\
= & {\left[\sum_{k=1}^{t} i_{1, k} \cdots i_{r-1, k}\left(1+\cdots+n_{r}\right)\right]+j_{1} \cdots j_{r-1} n_{r} } \\
& -\left[\sum_{k=1}^{t} n_{1} \cdots n_{r-2}\left(n_{r-1}-k+1\right)\left(1+\cdots+n_{r}\right)\right]-n_{1} \cdots n_{r-2}\left(n_{r-1}-t\right) n_{r} .
\end{aligned}
$$

By (3), the sequences $\left(i_{1, k}, \ldots, i_{r-1, k}\right)$ and $\left(n_{1}, \ldots, n_{r-2}, n_{r-1}-k+1\right)$ satisfy the conditions of Lemma 3.10, and, by (4), so do $\left(j_{1}, \ldots, j_{r-1}\right)$ and $\left(n_{1}, \ldots, n_{r-2}\right.$, $\left.n_{r-1}-t\right)$. Then the desired inequality $\beta_{r-1,2 r}(L) \geq \beta_{r-1,2 r}(J)$ follows from Lemma 3.10.

Example 3.11 Let $A=K\left[x_{1}, x_{2}, x_{3}, y_{1}, y_{2}, y_{3}, z_{1}, z_{2}, z_{3}\right]$ and $Q=\left(x_{1}, x_{2}, x_{3}\right)^{2}+$ $\left(y_{1}, y_{2}, y_{3}\right)^{2}+\left(z_{1}, z_{2}, z_{3}\right)^{2}$. Let

$$
L=\left(x_{3} y_{3}, x_{3} z_{3}, x_{3} y_{2} z_{2}\right)+Q
$$

and

$$
I=\left(x_{3} y_{3}, x_{3} y_{2}\right)+Q .
$$

Then, $L$ is the lex-plus- $Q$ ideal having the same Hilbert function as $I$. The following are betti diagrams of these ideals computed by Macaulay 2 .

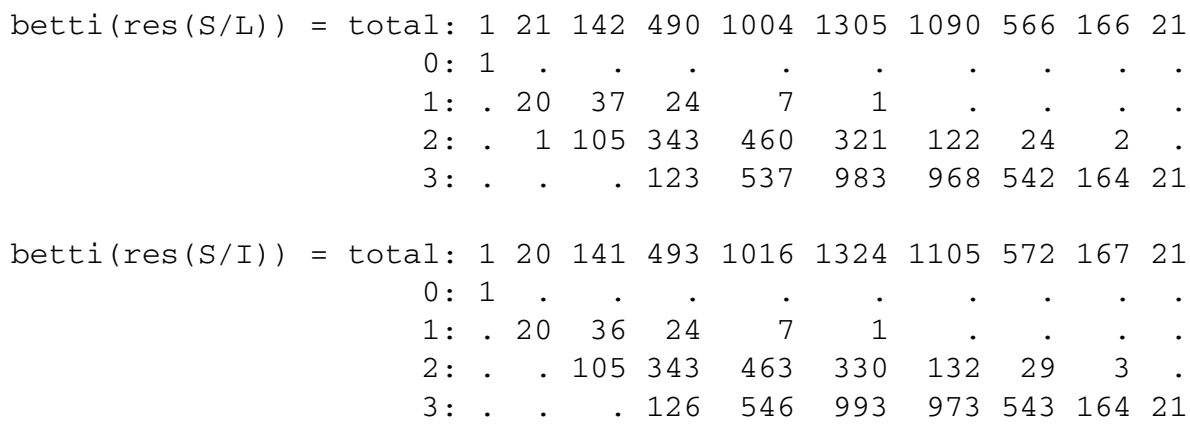




\section{Betti numbers of Borel ideals over a colored squarefree ring}

In the previous section, we saw that most colored squarefree rings do not satisfy Conjecture 1.2(2). On the other hand, we are not sure whether Conjecture 1.2(1) fails for those rings. Indeed, the ideals used in the proof of Theorem 3.5 do not give a counterexample to Conjecture 1.2(1). The purpose of this section is to give a way to compute the graded Betti numbers of lex ideals of a colored squarefree ring, which will be the fist step in studying Conjecture 1.2(1) for colored squarefree rings.

Let $R=S / Q$ be an $r$-colored squarefree ring as defined in Definition 1.3. For a finitely generated graded $R$-module $M$, let $\beta_{i, j}^{R}(M)$ and $\beta_{i}^{R}(M)$ be the graded Betti numbers of $M$ and the total Betti numbers of $M$ over $R$ respectively. A homogeneous ideal $I$ of $R$ has a linear resolution if there exists an integer $d$ such that $\beta_{i, i+j}^{R}(I)=0$ for all $i$ if $j \neq d$.

Lemma 4.1 Let $A=K\left[x_{1}, \ldots, x_{n}\right], P=\left(x_{1}, \ldots, x_{n}\right)^{2}$ and $B=A / P$. Then the ideal $I=\left(x_{1}, \ldots, x_{p}\right)$ of $B$ has a linear resolution and $\beta_{i}^{B}(I)=p n^{i}$ for all $i$.

Proof It is clear that the first syzygy module of the ideal $\left(x_{1}\right)$ of $B$ is $\left(x_{1}, \ldots, x_{n}\right)$. Since $I=\left(x_{1}\right) \bigoplus_{B}\left(x_{2}\right) \bigoplus_{B} \cdots \bigoplus_{B}\left(x_{p}\right)$, the first syzygy module of $I$ is isomorphic to $\left(x_{1}, \ldots, x_{n}\right) \bigoplus_{B} \cdots \bigoplus_{B}\left(x_{1}, \ldots, x_{n}\right) \subset B^{p}$. Then $I$ has a linear resolution and $\beta_{i+1}(I)=n \beta_{i}(I)$ for all $i \geq 0$.

For a subset $W \subset V$, write $I_{W}$ for the ideal of $R$ generated by $W$.

Lemma 4.2 Let $W \subset V$ and $p_{j}=\left|W \cap V_{j}\right|$. Then $I_{W}$ has a linear resolution and

$$
\beta_{i}^{R}\left(I_{W}\right)=\sum_{i_{1}+\cdots+i_{r}=i}\left(n_{1}^{i_{1}} \cdots n_{r}^{i_{r}}\right)\left(\prod_{i_{k} \neq 0} p_{k}\right) \text { for all } i .
$$

Proof Let $I_{\{k\}}$ be the ideal of $R_{\{k\}}=K\left[V_{k}\right] /\left(x_{k, 1}, \ldots, x_{k, n_{k}}\right)^{2}$ generated by $W \cap V_{k}$. Let $\mathbf{F}_{\{k\}}$ be the minimal free resolution of $R_{\{k\}} / I_{\{k\}}$ over $R_{\{k\}}$. Since

$$
R=R_{\{1\}} \otimes_{K} R_{\{2\}} \otimes_{K} \cdots \otimes_{K} R_{\{r\}}
$$

and

$$
R / I_{W}=\left(R_{\{1\}} / I_{\{1\}}\right) \otimes_{K}\left(R_{\{2\}} / I_{\{2\}}\right) \otimes_{K} \cdots \otimes_{K}\left(R_{\{r\}} / I_{\{r\}}\right),
$$

by the Künneth tensor formula, $\mathbf{F}_{\{1\}} \otimes_{K} \cdots \otimes_{K} \mathbf{F}_{\{r\}}$ is the minimal free resolution of $R / I_{W}$ over $R$. This fact and Lemma 4.1 prove the desired formula.

Definition 4.3 For monomials $u=y_{1} \cdots y_{t}$ and $v=z_{1} \cdots z_{t}$ of $R$ of the same degree, where $y_{1}>\cdots>y_{t}$ and $z_{1}>\cdots>z_{t}$ are variables, write $u \geq_{P} v$ if $y_{k} \geq z_{k}$ for all $k$. A monomial ideal $I$ of $R$ is said to be Borel if, for all monomials $u, v \in R, u \in I$ and $v>_{P} u$ implies $v \in I$. 
Note that lex ideals are Borel. For all monomials $u, v \in R$, write $u \succ v$ if $\operatorname{deg} u<\operatorname{deg} v$ or $\operatorname{deg} u=\operatorname{deg} v$ and $u>_{\operatorname{lex}} v$. Let $G(I)$ be the set of minimal monomial generators of a monomial ideal $I \subset R$.

Lemma 4.4 Let $I \subset R$ be a Borel ideal with $G(I)=\left\{u_{1}, \ldots, u_{t}\right\}$, where $u_{1} \succ \cdots \succ$ $u_{t}$. Then, for $k=1,2, \ldots, t$,

$$
\left(\left(u_{1}, \ldots, u_{k-1}\right): u_{k}\right)=\left(\left\{y \in V: y>\operatorname{last}\left(u_{k}\right) \text { or } \operatorname{color}(y) \subset \operatorname{color}\left(u_{k}\right)\right\}\right),
$$

where $\left(u_{1}, \ldots, u_{k-1}\right)=0$ if $k=1$.

Proof Since $I$ is Borel, it is clear that the left-hand side contains the right-hand side. We show that the right-hand side contains the left-hand side.

Let $f u_{k} \in\left(u_{1}, \ldots, u_{k-1}\right)$ be a monomial and $d=\operatorname{deg} u_{k}$. If $f u_{k}=0$ then there exists a monomial $y \in V_{a}$ with $a \in \operatorname{color}\left(u_{k}\right)$ such that $y$ divides $f$. Suppose $f u_{k} \neq 0$. Write $f u_{k}=y_{1} \cdots y_{s}$, where $y_{1}>\cdots>y_{s}$ are variables. Since $\left(u_{1}, \ldots, u_{k-1}\right)$ is Borel, there exists an integer $1 \leq \delta \leq d$ such that $y_{1} \cdots y_{\delta} \in G(I)$. Let $u_{p}=y_{1} \cdots y_{\delta}$. Then last $\left(u_{p}\right)>\operatorname{last}\left(u_{k}\right)$ and $u_{p}$ does not divide $u_{k}$. Hence there exists $y_{\ell}$ which divides $u_{p}$ but does not divide $u_{k}$. This $y_{\ell}$ must divide $f$, and $y_{\ell}>\operatorname{last}\left(u_{k}\right)$.

For any monomial $m \in R$, let

$$
W(m)=\{y \in V: y<\operatorname{last}(m) \text { and } \operatorname{color}(y) \not \subset \operatorname{color}(m)\}
$$

and

$$
T(m)=V \backslash W(m)=\{y \in V: y>\operatorname{last}(m) \text { or } \operatorname{color}(y) \subset \operatorname{color}(m)\} .
$$

Proposition 4.5 Let $I \subset R$ be Borel. Then

$$
\beta_{i, i+j}^{R}(I)=\sum_{u \in G(I), \operatorname{deg}(u)=j} \beta_{i}^{R}\left(R / I_{T(u)}\right) \quad \text { for all } i, j .
$$

Proof The proof is the same as that of [10, Lemma 1.5]. Let $G(I)=\left\{u_{1}, \ldots, u_{t}\right\}$ with $u_{1} \succ \cdots \succ u_{t}$. We use induction on $t$. If $t=1$ then the first syzygy module of $I=\left(u_{1}\right)$ is $\left(0: u_{1}\right)=I_{T\left(u_{1}\right)}$, and therefore the statement follows from Lemma 4.2.

Suppose $t>1$. Let $J=\left(u_{1}, \ldots, u_{t-1}\right)$ and $d=\operatorname{deg} u_{t}$. Note that $J$ is also Borel. Consider the short exact sequence

$$
0 \longrightarrow R /\left(J: u_{t}\right)(-d) \stackrel{\times u_{t}}{\longrightarrow} R / J \longrightarrow R / I \longrightarrow 0 .
$$

By Lemma 4.4, $\left(J: u_{t}\right)=I_{T\left(u_{t}\right)}$. Let $\mathbf{G}$ be the minimal free resolution of $R / J$ over $R$ and $\mathbf{F}$ the minimal free resolution of $R /\left(J: u_{t}\right)(-d)=R / I_{T\left(u_{t}\right)}(-d)$. One has $\mathbf{G}_{i}=\bigoplus_{j \in \mathbb{N}} R(-j)^{\beta_{i, j}^{R}(R / J)}$. By induction, $\max \left\{j: \beta_{i, j}^{R}(R / J) \neq 0\right\} \leq i+d-1$. On the other hand, by Lemma $4.2 \mathbf{F}_{i}=R(-d-i)^{\beta_{i}^{R}\left(R / I_{T\left(u_{t}\right)}\right)}$. Hence, $R / I$ is minimally resolved by the mapping cone arising from the map $R /\left(J: u_{t}\right)(-d) \stackrel{\times u_{t}}{\longrightarrow} R / J$. The statement follows. 
Corollary 4.6 The graded Betti numbers of Borel ideals of $R$ do not depend on the characteristic of the base field $K$.

Remark 4.7 The key idea in the proof of Proposition 4.5 is that the colon ideal $\left(\left(u_{1}, \ldots, u_{k-1}\right): u_{k}\right)$ has a 1-linear resolution. This property also holds for Borel ideals in the quotient ring $S / M$, whenever $M$ is an ideal generated by monomials of degree 2. Thus, the Betti numbers of Borel ideals of such rings can be computed in the same way as in the proof of Proposition 4.5.

One might think that the graded Betti numbers of a Borel ideal $I=\left(u_{1}, \ldots, u_{t}\right)$ depend on the shape of $T\left(u_{j}\right)$. We will show that they only depend on $\operatorname{grow}\left(u_{j}\right)=$ $\left|W\left(u_{j}\right)\right|$. For an integer $0 \leq a \leq\left|\bigcup_{j=d+1}^{r} V_{j}\right|$, let $W(a, d)$ be the set of lex-smallest variables in $\bigcup_{j=d+1}^{r} V_{j}$ with $|W(a, d)|=a$. Set $T(a, d)=V \backslash W(a, d)$.

Lemma 4.8 Let $m \in R$ be a monomial of degree $d$ with $\operatorname{grow}(m)=a$. Then $a \leq$ $\left|\bigcup_{j=d+1}^{r} V_{j}\right|$ and $I_{T(m)}$ has the same graded Betti numbers as $I_{T(a, d)}$.

Proof For any subset $W \subset V$, let $R[W]=K[W] /(Q \cap K[W]) \cong R / I_{V \backslash W}$. By Lemma 4.2 , we must prove that there exists a subset $\tilde{W}$ such that $R[\tilde{W}]$ has the same Hilbert function as $R[W(m)]$ and $\tilde{W}$ is a set of lex-smallest variables in $\bigcup_{j=d+1}^{r} V_{j}$.

For a subset $W \subset V$, let $c_{i}(W)=\left|W \cap V_{i}\right|$ for $i=1,2, \ldots, r$ and $\mathbf{c}(W)=$ $\left(c_{1}(W), \ldots, c_{r}(W)\right)$. Note that the Hilbert function of $R[W]$ only depends on the vector $\mathbf{c}(W)$. Let $e_{i}=\left|W(m) \cap V_{i}\right|$ for $i=1,2, \ldots, r$ and $\mathbf{e}=\left(e_{1}, \ldots, e_{r}\right)$. Let $\operatorname{last}(m)=x_{s, t}$ and $q=\max \left\{j: n_{j} \geq t\right\}$. Then, by (1) (in the proof of Lemma 2.10), the vector $\mathbf{e}$ can be written in the form

$$
\mathbf{e}=\left(e_{1}, \ldots, e_{q}, n_{q+1}, \ldots, n_{r}\right)
$$

and each $e_{k}$ is either $0, t-1$ or $t$ for $k=1,2, \ldots, q$. Consider the vector of the form

$$
\tilde{\mathbf{e}}=\left(\tilde{e}_{1}, \ldots, \tilde{e}_{r}\right)=\left(0, \ldots, 0, t-1, \ldots, t-1, t, \ldots, t, n_{q+1}, \ldots, n_{r}\right)
$$

which is obtained by a permutation of entries of e. Let $\tilde{W}=\bigcup_{i=1}^{r}\left\{x_{i, j}: 1 \leq j \leq \tilde{e}_{i}\right\}$. Then $\mathbf{c}(\tilde{W})=\tilde{\mathbf{e}}$. By the construction of $\tilde{\mathbf{e}}$, it follows that $R[\tilde{W}]$ and $R[W(m)]$ have the same Hilbert function. Also, by the formula of $\tilde{\mathbf{e}}$, it is clear that $\tilde{W}$ is a set of lex-smallest variables in $\bigcup_{j=d+1}^{r} V_{j}$.

By Proposition 4.5 and Lemma 4.8, we get

Theorem 4.9 Let $I=\left(u_{1}, \ldots, u_{t}\right)$ be a Borel ideal of $R$ with grow $\left(u_{k}\right)=a_{k}$. Then

$$
\beta_{i, i+j}^{R}(I)=\sum_{u_{k} \in G(I), \operatorname{deg} u_{k}=j} \beta_{i}^{R}\left(R / I_{T\left(a_{k}, j\right)}\right) \quad \text { for all } i, j .
$$


Example 4.10 Let $I, L$ and $R=S / Q$ be as in Example 3.11. Let $\tilde{I}=\left(x_{3} y_{3}, x_{3} y_{2}\right)$ and $\tilde{L}=\left(x_{3} y_{3}, x_{3} z_{3}, x_{3} y_{2} z_{2}\right)$ be ideals of $R$. Since $L$ is lex-plus- $Q, \tilde{L}$ is lex. Also $W\left(x_{3} y_{3}\right)=\left\{z_{1}, z_{2}, z_{3}\right\}, W\left(x_{3} z_{3}\right)=\left\{y_{1}, y_{2}\right\}$ and $W\left(x_{3} y_{2} z_{2}\right)=\emptyset$. Hence, by Proposition 4.5,

$$
\begin{aligned}
\beta_{i, i+2}^{R}(\tilde{L}) & =\beta_{i}^{R}\left(R /\left(x_{1}, x_{2}, x_{3}, y_{1}, y_{2}, y_{3}\right)\right)+\beta_{i}^{R}\left(R /\left(x_{1}, x_{2}, x_{3}, y_{3}, z_{1}, z_{2}, z_{3}\right)\right) \\
& =(i+1) 3^{i}+\left\{(i+1) 3^{i}+\left(\begin{array}{c}
i+1 \\
2
\end{array}\right) 3^{i-1}\right\}
\end{aligned}
$$

and

$$
\beta_{i, i+3}^{R}(\tilde{L})=\beta_{i}^{R}\left(R /\left(x_{1}, x_{2}, x_{3}, y_{1}, y_{2}, y_{3}, z_{1}, z_{2}, z_{3}\right)\right)=\left(\begin{array}{c}
i+2 \\
2
\end{array}\right) 3^{i} .
$$

Note that the ideal $\tilde{I}$ has a linear resolution and $\beta_{i, i+2}^{R}(\tilde{I})=2(i+1) 3^{i}$ for all $i$.

\section{Concluding remarks and open problems}

\subsection{Betti numbers of lex ideals}

As we show in Section 3, Conjecture 1.2(2) is false. However Conjecture 1.2(1) is still open even for colored squarefree rings.

Problem 5.1 Do lex ideals of a colored squarefree ring $R$ have the greatest graded Betti numbers among all homogeneous ideals of $R$ having the same Hilbert function?

While we give a combinatorial way to compute the graded Betti numbers of Borel ideals of colored squarefree rings, we do not know whether lex ideals have the greatest graded Betti numbers among all Borel ideals having the same Hilbert function. The only known case is

Proposition 5.2 Lex ideals of a 2-colored squarefree ring $R$ have the greatest graded Betti numbers among all homogeneous ideals of $R$ having the same Hilbert function.

Proof Let $I=\left(u_{1}, \ldots, u_{s}, v_{1}, \ldots, v_{t}\right)$ be a monomial ideal of $R$ with $\operatorname{deg} u_{k}=1$ and $\operatorname{deg} v_{\ell}=2$. Then $I=\left(u_{1}, \ldots, u_{s}\right) \oplus_{R}\left(v_{1}\right) \oplus_{R} \cdots \oplus_{R}\left(v_{t}\right)$. Then, by Lemma 4.2,

$$
\beta_{i, i+2}^{R}(I)=\sum_{j=1}^{t} \beta_{i, i+2}^{R}\left(\left(v_{j}\right)\right)=t \beta_{i}^{R}(R / \mathfrak{m}) \text { for all } i,
$$

where $\mathfrak{m}$ is the maximal ideal of $R$, and $\beta_{i, i+j}^{R}(I)=0$ if $j \neq 1,2$.

Let $L=\left(u_{1}^{\prime}, \ldots, u_{s}^{\prime}, v_{1}^{\prime}, \ldots, v_{t^{\prime}}^{\prime}\right)$ be the lex ideal of $R$ having the same Hilbert function as $I$, where $\operatorname{deg} u_{k}^{\prime}=1$ and $\operatorname{deg} v_{\ell}^{\prime}=2$. Since $L$ is lex, $t^{\prime} \geq t$. Hence $\beta_{i, i+2}^{R}(L)=t^{\prime} \beta_{i}^{R}(R / \mathfrak{m}) \geq \beta_{i, i+2}^{R}(I)$ for all $i$. Since $I$ and $L$ have the same Hilbert 
function, we have $\sum_{i}(-1)^{i} \beta_{i, j}^{R}(I)=\sum_{i}(-1)^{i} \beta_{i, j}^{R}(L)$ for all $j$. Since $\beta_{i, i+j}^{R}(I)=$ $\beta_{i, i+j}^{R}(L)=0$ if $j \neq 1,2$, it follows that

$$
\beta_{j-1, j}^{R}(L)-\beta_{j-2, j}^{R}(L)=\beta_{j-1, j}^{R}(I)-\beta_{j-2, j}^{R}(I) .
$$

Thus we have $\beta_{i, i+1}^{R}(L) \geq \beta_{i, i+1}^{R}(I)$ for all $i$ as desired.

\subsection{Betti numbers of Stanley-Reisner ideals}

One may ask the following question which is similar to Problem 5.1: Does there always exist an $r$-colored complex whose Stanley-Reisner ideal has the greatest graded Betti numbers among all $r$-colored complexes for a fixed face vector?

Unfortunately, the answer is no. Indeed, 2-colored complexes are bipartite graphs, and if we consider bipartite graphs with 10 edges and 7 vertices (there are only 4 such bipartite graphs up to a permutation of the vertices), then computer computations show that none of them have the greatest graded Betti numbers.

\section{$5.3 h$-vectors of balanced Cohen-Macaulay complexes}

A further generalization of Theorem 2.1 is the next problem.

Problem 5.3 Let $S=K[V]$ and $Q=\sum_{j=1}^{r}\left(x_{j, 1}, \ldots, x_{j, n_{j}}\right)^{a_{j}}$, where $a_{1}, \ldots, a_{r}$ are positive integers. Characterize the Hilbert functions of homogeneous ideals of $S / Q$.

Unfortunately, the above ring $S / Q$ is generally not Macaulay-Lex with respect to any order of the variables. However, Problem 5.3 would be interesting since it yields the complete description of $h$-vectors of balanced Cohen-Macaulay complexes (see $[3,24])$.

Acknowledgements We would like to thank Ryota Okazaki for useful discussions regarding Section 4 . The results and examples presented in this paper have been inspired by computations performed by the computer algebra system CoCoA [5] and Macaulay 2 [9].

\section{References}

1. Babson, E., Novik, I.: Face numbers and nongeneric initial ideals. Electron. J. Combin. 11 (2004/2006). Research Paper 25, 23 pp.

2. Bigatti, A.: Upper bounds for the Betti numbers of a given Hilbert function. Comm. Algebra 21, 2317-2334 (1993)

3. Björner, A., Frankl, P., Stanley, R.P.: The number of faces of balanced Cohen-Macaulay complexes and a generalized Macaulay theorem. Combinatorica 7, 23-34 (1987)

4. Clements, G.F., Lindström, B.: A generalization of a combinatorial theorem of Macaulay. J. Combinatorial Theory 7, 230-238 (1969)

5. CoCoA Team: CoCoA: a system for doing Computations in Commutative Algebra. Available at http://cocoa.dima.unige.it

6. Eisenbud, D.: Commutative algebra with a view toward algebraic geometry. Grad. Texts in Math., vol. 150. Springer, New York (1995)

7. Francisco, C.A., Richert, B.P.: Lex-plus-powers ideals. In: Peeva, I. (ed.) Syzygies and Hilbert functions. Lect. Notes Pure Appl. Math., vol. 254, pp. 113-144. CRC Press, Boca Raton (2007) 
8. Frankl, P., Füredi, Z., Kalai, G.: Shadows of colored complexes. Math. Scand. 63, 169-178 (1988)

9. Grayson, D., Stillman, M.: Macaulay 2, a software system for research in algebraic geometry. Available at http://www.math.uiuc.edu.Macaulay2/

10. Herzog, J., Takayama, Y.: Resolutions by mapping cones. Homology Homotopy Appl. 4, 277-294 (2002)

11. Hochster, M.: Cohen-Macaulay rings, combinatorics, and simplicial complexes. In: McDonald, B.R., Morris, R. (eds.) Ring theory, II, Proc. Second Conf., Univ. Oklahoma, Norman, Oklahoma. Lecture Notes in Pure and Appl. Math., vol. 26, pp. 171-223. Dekker, New York (1977)

12. Hulett, H.: Maximum Betti numbers of homogeneous ideals with a given Hilbert function. Comm. Algebra 21, 2335-2350 (1993)

13. London, E.: A new proof of the colored Kruskal-Katona theorem. Discrete Math. 126, 217-223 (1994)

14. Macaulay, F.S.: Some properties of enumeration in the theory of modular systems. Proc. London Math. Soc. 26, 531-555 (1927)

15. Mermin, J.: Compressed ideals. Bull. London Math. Soc. 40, 77-87 (2008)

16. Mermin, J., Murai, S.: The lex-plus-powers conjecture holds for pure powers (2008, submitted)

17. Mermin, J., Peeva, I.: Lexifying ideals. Math. Res. Letters 13, 409-422 (2006)

18. Mermin, J., Peeva, I.: Hilbert functions and lex ideals. J. Algebra 313, 642-656 (2007)

19. Mermin, J., Peeva, I., Stillman, M.: Ideals containing the squares of the variables. Adv. Math. 217, 2206-2230 (2008)

20. Murai, S.: Borel-plus-powers monomial ideals. J. Pure Appl. Algebra 212, 1321-1336 (2008)

21. Murai, S.: Betti numbers of strongly color-stable ideals and squarefree strongly color-stable ideals. J. Algebraic Combin. 27, 383-398 (2008)

22. Murai, S., Peeva, I.: Hilbert schemes and Betti numbers over a Clements-Lindström ring (2008, submitted)

23. Pardue, K.: Deformation classes of graded modules and maximal Betti numbers. Illinois J. Math. 40, 564-585 (1996)

24. Stanley, R.P.: Balanced Cohen-Macaulay complexes. Trans. Amer. Math. Soc. 249, 139-157 (1979) 